\title{
ON THE SOLUTIONS OF THE HEAT EQUATION ${ }^{1}$
}

\author{
W. FULKS
}

1. Introduction. In his Cours d'analyse mathématique, Goursat $[6, \text { p. 320 }]^{2}$ points out (and it had probably been noticed before) that the basic integral representations for solutions of the heat equation

$$
u_{x x}(x, t)-u_{t}(x, t)=0
$$

and for the solutions of Laplace's equation

$$
u_{x x}(x, t)+u_{t t}(x, t)=0
$$

have many similar properties. This analogy has recently served as a basis for an attack upon the properties of the solutions of (1.1), the basic properties of those of (1.2) being relatively well known. The first major step in this direction was made by D. V. Widder [11] who obtained a Stieltjes integral representation theorem for positive solutions of (1.1) in a half-plane or strip $0<t<B \leqq \infty$ which, as he remarked, is analogous to the corresponding representation given by Herglotz [8] and Riesz [10] for positive functions harmonic in the unit circle.

In 1950 Hartman and Wintner [7] published a paper in which they obtained a Stieltjes integral representation theorem for solutions of (1.1) in a rectangle. From this they drew many interesting and illuminating conclusions. However, their treatment of the representation of solutions of (1.1) by means of a Lebesgue integral leaves something to be desired as they effectively consider only bounded functions. The present author [5], in a University of Minnesota thesis in 1949, independently obtained the main results of Hartman and Wintner. In this thesis there are many more results which Hartman and Wintner do not give. There is a rather complete discussion of the representability of the solutions of (1.1) by a Lebesgue integral, in fact there are two sets of necessary and sufficient conditions for such representability. The purpose of this paper is to present one of these theorems.

Let $R$ be a rectangle $0<x<1,0<t<B \leqq \infty$, and let us denote by $H$ the class of functions which satisfy (1.1) and for which $u_{x x}$ and $u_{t}$

Received by the editors November 2, 1950 .

${ }^{1}$ This paper is, in essence, a part of a thesis written under the direction of Professor S. E. Warschawski. The author wishes to express his most grateful thanks to Professor Warschawski for his sincere and excellent guidance.

${ }^{2}$ Numbers in brackets refer to the bibliography at the end of the paper. 
are continuous. Let $G(x, t ; \xi, \tau)$ denote the function

$$
\frac{1}{2}\left[\vartheta_{3}\left(\frac{x-\xi}{2}, t-\tau\right)-\vartheta_{3}\left(\frac{x+\xi}{2} \cdot t-\tau\right)\right]
$$

where $\vartheta_{3}$ is the Jacobi theta function. Then, though their statements are formulated differently, Hartman and Wintner prove the following:

Theorem A. For $u(x, t)$ to be representable in $R$ by

$$
\begin{aligned}
u(x, t)= & \int_{0+}^{1-0} G(x, t ; \xi, 0) d \alpha(\xi)+\int_{0}^{t} G_{\xi}(x, t ; 0, \tau) d \beta(\tau) \\
& -\int_{0}^{t} G_{\xi}(x, t ; 1, \tau) d \gamma(\tau)
\end{aligned}
$$

where $\alpha(\xi)$ is of bounded variation in every closed interval in $0<\xi<1$ and $\beta(\tau), \gamma(\tau)$ are of bounded variation in every closed interval of $0 \leqq \tau$ $<B \leqq \infty$ it is necessary and sufficient that:

(1) $u(x, t) \in H$ in $R$.

(2) $\int_{0}^{t} u(\delta, \tau) d \tau$ and $\int_{0}^{t} u(1-\delta, \tau) d \tau, 0 \leqq t<B$, be of uniform bounded variation $0<\delta \leqq \delta_{0}$ for some $\delta_{0}>0$ in every closed interval of $0 \leqq t<B$ $\leqq \infty$.

(3) $\int_{0}^{1} \xi(1-\xi)|u(\xi, \delta)| d \xi \leqq M, 0<\delta \leqq \delta_{0}$, where $M$ is a constant.

They further establish:

THEOREM B. For any function representable in the form (1.3) in $R$, the normal limits at the boundary are given by

$$
\lim _{t \rightarrow 0+} u(x, t)=\alpha^{\prime}(x), \quad \lim _{x \rightarrow 0+} u(x, t)=\beta^{\prime}(t), \quad \lim _{x \rightarrow 1-0} u(x, t)=\gamma^{\prime}(t)
$$

wherever the derivatives in question exist.

These results are also given in [5].

2. Elementary properties of the kernel function. The kernel function in (1.3) is given by the equation

$$
G(x, t ; \xi, \tau)=\frac{1}{2}\left[\vartheta_{3}\left(\frac{x-\xi}{2}, t-\tau\right)-\vartheta_{3}\left(\frac{x+\xi}{2}, t-\tau\right)\right]
$$

where $\vartheta_{3}$ is the well known Jacobi theta function. Two convenient formulas for $\vartheta_{3}$ are

$$
\vartheta_{3}\left(\frac{1}{2} x, t\right)=1+\sum_{n=1}^{\infty} \cos n \pi x \exp \left[-n^{2} \pi^{2} t\right]
$$


and

$$
\vartheta_{3}\left(\frac{1}{2} x, t\right)=(\pi t)^{-1 / 2} \sum_{n=-\infty}^{\infty} \exp \left[-(x+2 n)^{2} / 4 t\right]
$$

(See Doetsch [1, pp. 26 and 307].) It is easily seen from these two representations for $\vartheta_{3}$ that $G(x, t ; \xi, \tau)$ and its derivative $G_{\xi}(x, t ; \xi, \tau)$ are uniformly continuous functions as long as $(x, t)$ is bounded away from $(\xi, \tau)$.

REMARKS. 1. From a further representation

$$
\vartheta_{3}\left(\frac{1}{2} x, t\right)=Q_{0} Q_{2}^{2} \prod_{n=1}^{\infty}\left[1-\left(\sin ^{2} \frac{1}{2} \pi x / \cosh ^{2}\left(n-\frac{1}{2}\right) \pi^{2} t\right)\right]
$$

(see Magnus and Oberhettinger [9, p. 141]) where the $Q$ 's are positive functions of $t$ for $t>0$, it is clear that

$$
\begin{array}{lll}
G_{\xi}(x, t ; 0, \tau)>0 & \text { for } 0<x<1 \quad \text { and } t>\tau, \\
G_{\xi}(x, t ; 1, \tau)<0 & \text { for } 0<x<1 & \text { and } t>\tau, \\
G(x, t ; \xi, \tau)>0 & \text { for } 0<x, \xi<1, & \text { and } t>\tau .
\end{array}
$$

2. For fixed $x, t, 0<x<1, \epsilon \leqq t-\tau \leqq B^{\prime}$, where $\epsilon, B$ are two positive constants, there exist three positive constants $c, C$, and $\delta$, which depend only upon $x, t, \epsilon$, and $B^{\prime}$ such that

$$
\begin{array}{rlrl}
C_{\xi} & \geqq G(x, t ; \xi, \tau) \geqq c \xi, & 0<\xi<\delta, \\
C(1-\xi) \geqq G(x, t ; \xi, \tau) \geqq c(1-\xi), & 0<1-\xi<\delta .
\end{array}
$$

The proofs of these remarks are indicated on p. 380 of the paper by Hartman and Wintner.

3. The Lebesgue integral representation. We are now prepared to state and prove our basic

TheOREM. For $u(x, t)$ to be representable in $R$ by

$$
\begin{aligned}
u(x, t)= & \int_{0}^{1} G(x, t ; \xi, 0) a(\xi) d \xi+\int_{0}^{t} G_{\xi}(x, t ; 0, \tau) b(\tau) d \tau \\
& -\int_{0}^{t} G_{\xi}(x, t ; 1, \tau) c(\tau) d \tau
\end{aligned}
$$

where $a(\xi) \in L$ in every closed interval in $0<\xi<1, b(\tau), c(\tau) \in L$ in every closed interval in $0 \leqq \tau<B \leqq \infty$, it is necessary and sufficient that

$$
u(x, t) \in H \text { in } R \text {. }
$$




$$
\begin{aligned}
& \lim _{\delta \rightarrow 0, \delta^{\prime} \rightarrow 0} \int_{0}^{t}\left|u(\delta, \tau)-u\left(\delta^{\prime}, \tau\right)\right| d \tau=0, \\
& \lim _{\delta \rightarrow 0, \delta^{\prime} \rightarrow 0} \int_{0}^{t}\left|u(1-\delta, \tau)-u\left(1-\delta^{\prime}, \tau\right)\right| d \tau=0
\end{aligned}
$$

for every $t, 0<t<B \leqq \infty$.

$$
\lim _{\delta \rightarrow 0, \delta^{\prime} \rightarrow 0} \int_{0}^{1} \xi(1-\xi)\left|u(\xi, \delta)-u\left(\xi, \delta^{\prime}\right)\right| d \xi=0 .
$$

It might be pointed out that this gives us immediately a result on the uniqueness of the solution of the boundary value problem associated with (1.1) in $R$ as a

CoRollary. If $u(x, t)$ satisfies conditions (1), (2), and (3) and in addition $u(x, 0+)=0, u(0+, t)=0, u(1-0, t)=0$ almost everywhere, then $u(x, t) \equiv 0$ in $R$.

For $u(x, t)$ is representable by (3.1), and by Theorem B, the functions $a, b$, and $c$ vanish almost everywhere.

This seems to be an improvement over known results. A résumé of those obtained prior to 1936 is contained in Doetsch [2]. Typical of these is the result of Volterra quoted on p. 56 which requires in addition that both $u$ and $u_{x}$ be continuous in the "closed" rectangle $0 \leqq x \leqq 1,0 \leqq t<B \leqq \infty$.

We now proceed to prove the sufficiency of the conditions of the theorem. Let the closed interval $I \subset\{0 \leqq t<B \leqq \infty\}$ be prescribed and $e$ be any measurable set in $I$ :

$$
\int_{e}|u(\delta, \tau)| d \tau-\int_{e}\left|u\left(\delta^{\prime}, \tau\right)\right| d \tau \leqq \int_{0}\left|u(\delta, \tau)-u\left(\delta^{\prime}, \tau\right)\right| d \tau .
$$

But given $\epsilon>0$, there exists $\eta(\epsilon, I)$ such that

$$
\int_{I}\left|u(\delta, \tau)-u\left(\delta^{\prime}, \tau\right)\right| d \tau \leqq \epsilon / 2 \text { for } \delta, \delta^{\prime}<\eta(\epsilon, I)
$$

Then

$$
\int_{0}|u(\delta, \tau)| d \tau \leqq \int_{0}\left|u\left(\delta^{\prime}, \tau\right)\right| d \tau+\epsilon / 2 .
$$

Now keep $\delta^{\prime}$ fixed: by the property of absolute continuity of an integral we can take $m(e)$ so small that the integral on the right is bounded by $\epsilon / 2$. Hence 


$$
\int_{e}|u(\delta, \tau)| d \tau<\epsilon
$$

uniformly for $0<\delta<\eta(\epsilon, I)$, for $m(e)$ sufficiently small. This establishes uniform absolute continuity of $\int_{0}^{t} u(\delta, \tau) d \tau$ in any closed interval of $0 \leqq t<B \leqq \infty$. Hence by formula $\left(15_{1}\right)$ on p. 374 of Hartman and Wintner, $d \beta(\tau)$ in (1.3) can be replaced by $b(\tau) d \tau$. Similarly $d \gamma(\tau)$ can be replaced by $c(\tau) d \tau$. By $\left(15_{3}\right)$ of Hartman and Wintner, $d \alpha(\xi)$ can be replaced by $a(\xi) d \xi$ in any closed interior interval of $0<\xi<1$. Hence, passing to the limits,

$$
\int_{0+}^{1-0} G(x, t ; \xi, 0) d \alpha(\xi)=\int_{0}^{1} G(x, t ; \xi, 0) a(\xi) d \xi
$$

since the integral converges absolutely.

We turn next to the necessity of our conditions. We shall first assume that $u(x, t) \geqq 0$ in $R$, then later remove this restriction.

By Hartman and Wintner formula $\left(15_{1}\right)$

$$
\lim _{x \rightarrow 0+} \int_{0}^{t} u(x, \tau) d \tau=\int_{0}^{t} b(\tau) d \tau
$$

and by Theorem $\mathrm{B}$, for almost all $t, 0 \leqq t<B \leqq \infty$, we have that $\lim _{x \rightarrow 0+} u(x, t)=b(t)$. These relations imply that

$$
\lim _{\delta \rightarrow 0} \int_{0}^{t}|u(\delta, \tau)-b(\tau)| d \tau=0 .
$$

[For if (i) $f_{n}(x) \geqq 0$, (ii) $\lim _{n \rightarrow \infty} f_{n}(x)=f(x)$ almost everywhere, $a \leqq x$ $\leqq b$, (iii) $\lim _{n \rightarrow \infty} \int_{a}^{b} f_{n}(x) d x=\int_{a}^{b} f(x) d x$, then $\lim _{n \rightarrow \infty} \int_{a}^{b}\left|f_{n}(x)-f(x)\right| d x=0$. This follows easily from a result of de la Vallée Poussin quoted by Evans [4, p. 13].]

To remove the restriction of positivity we note that $u(x, t)$ can be written as the difference of two positive functions. For we can write $a(x)=a_{1}(x)-a_{2}(x)$ where $a_{1}(x)$ and $a_{2}(x)$ are positive, for example, $a_{1}(x)=|a(x)|$ and $a_{2}(x)=|a(x)|-a(x)$, and we can similarly separate $b(t)$ and $c(t)$, and write $u(x, t)=u_{1}(x, t)-u_{2}(x, t)$ where $u_{1}(x, t)$ has the boundary values $a_{1}(x), b_{1}(t)$, and $c_{1}(t)$, and $u_{2}(x, t)$ has the boundary values $a_{2}(x), b_{2}(t)$, and $c_{2}(t)$, and where $u_{1}(x, t)$ and $u_{2}(x, t)$ are clearly non-negative functions by Remark 1 . By applying the above result to $u_{1}(x, t)$ and $u_{2}(x, t)$, we see that the result is true for $u(x, t)$ $=u_{1}(x, t)-u_{2}(x, t)$. Similarly the result is true for approach to the right side of the region $R$. But these results imply condition (2) of the theorem. 
To establish condition (3) we make use of the relation (see Doetsch $[3$, p. 615])

$$
\int_{0}^{1} G(y, \delta ; x, 0) G(x, t ; \xi, 0) d x=G(y, t+\delta ; \xi, 0) .
$$

Now from our representation (3.1) we obtain

$$
\begin{aligned}
\int_{0}^{1} G(y, \delta ; x, 0) u(x, t) d x & =\int_{0}^{1} G(y, \delta ; x, 0) \int_{0}^{1} G(x, t ; \xi, 0) a(\xi) d \xi d x \\
& +\int_{0}^{1} \int_{0}^{t} G(y, \delta ; x, 0) G_{\xi}(x, t ; 0, \tau) b(\tau) d \tau d x \\
& -\int_{0}^{1} \int_{0}^{t} G(y, \delta ; x, 0) G_{\xi}(x, t ; 1, \tau) c(\tau) d \tau d x
\end{aligned}
$$

Applying the dominated convergence theorem to the last two integrals as double integrals we see that they vanish as $t \rightarrow 0+$. The first integral of (3.4) can, by the use of (3.3), be written as

$$
\int_{0}^{1} G(y, t+\delta ; \xi, 0) a(\xi) d \xi .
$$

Then passing to the limit under the integral sign we get

$$
\lim _{t \rightarrow 0+} \int_{0}^{1} G(y, \delta ; x, 0) u(x, t) d x=\int_{0}^{1} G(y, \delta ; \xi, 0) a(\xi) d \xi .
$$

And by Theorem $\mathrm{B}$ we have, for almost all $x, 0<x<1$,

$$
\lim _{t \rightarrow 0+} u(x, t)=a(x)
$$

Hence, if $u(x, t)$ is positive, we can again invoke the theorem on strong and weak convergence to obtain

$$
\lim _{t \rightarrow 0+} \int_{0}^{1} G(y, \delta ; \xi, 0)|u(\xi, t)-a(\xi)| d \xi=0 .
$$

We can remove the condition of positivity as before. This then implies

$$
\lim _{t \rightarrow 0, t^{\prime} \rightarrow 0} \int_{0}^{1} G(y, \delta ; \xi, 0)\left|u(\xi, t)-u\left(\xi, t^{\prime}\right)\right| d \xi=0 .
$$

But by the second remark and the fact that $G(y, \delta ; \xi, 0)$ is bounded 
away from zero in any closed interval interior to $0<\xi<1$, this last equation is equivalent to condition (3) of the theorem.

\section{BIBLIOGRAPHY}

1. G. Doetsch, Theorie und Anwendung der Laplace-Transformation, New York, 1943.

2. - Les equations aux derivées partielles du type parabolique, l'Enseignement Mathematique vol. 35 (1936).

3. , Problem aus der Theorie der Warmleitung, III Mitteilung, Math. Zeit. vol. 25 (1926).

4. G. C. Evans, The logarithmic potential, New York, 1927.

5. W. Fulks, On integral representations and uniqueness of solutions of the heat equation, University of Minnesota Thesis, June, 1949.

6. E. Goursat, Cours d'analyse mathématique, vol. 3, Paris, 1927.

7. P. Hartman and A. Wintner, On the solutions of the equation of heat conduction, Amer. J. Math. vol. 72 (1950).

8. G. Herglotz, Über Potenzreihen mit positiven reelen Teil im Einheitskreis, Berichte über die Verhandlungen der honiglich Sachsischen Gesellschaft der Wissenschaften zu Leipzig, Mathematisch-physische Klasse vol. 63 (1911) pp. 6-9.

9. W. Magnus and F. Oberhettinger, Formeln und Sätze fïr die speziellen Funktionen der mathematischen Physik, Berlin and Heidelberg, 1948.

10. F. Riesz, Sur certains systèmes singuliers d'équation intégrales, Ann. Ecole Norm. (3) vol. 28 (1911).

11. D. V. Widder, Positive temperatures on an infinite rod, Trans. Amer. Math. Soc. vol. 55 (1944).

The University of Minnesota 